\title{
Efficacy and Safety of Surfrin Intranasal Spray, a Natural Phospholipid Spray, in Treating Patients With Acute Rhinitis: a Multicenter, Randomized, Controlled Trial
}

\section{Yunying Li ( $\nabla$ docliyunying@gzucm.edu.cn )}

The Second Affiliated Hospital of Guangzhou University of Chinese Medicine https://orcid.org/00000002-0424-0154

Jiyan Xia

The Second Affiliated Hospital of Guangzhou University of Chinese Medicine Qiulan Luo

The Second Affiliated Hospital of Guangzhou University of Chinese Medicine

\section{Peiyuan Wang}

The First Affiliated Hospital of Guangzhou University of Chinese Medicine

\section{Haobo Zou}

The Second Affiliated Hospital of Guangzhou University of Chinese Medicine

\section{Wei Huang}

The second clinical college of Guangzhou University of Chinese Medicine

\section{Xiaocong Feng}

The second clinical college of Guangzhou University of Chinese Medicine

\section{Jianhui Wang}

The first affiliated Hospital of Guangzhou University of Chinese Medicine

\section{Wenwei Ouyang}

The Second Affiliated Hospital of Guangzhou University of Chinese Medicine

\section{Yan Liang}

The Second Affiliated Hospital of Guangzhou University of Chinese Medicine

Chunsong Liu

the First Affiliated Hospital of Guangzhou University of Chinese Medicine

\section{Yingfang Wu}

The Second Affiliated Hospital of Guangzhou University of Chinese Medicine Chunqiao Li

the First Affiliated Hospital of Guangzhou University of Chinese Medicine

\section{Wenyong Chen}

The Second Affiliated Hospital of Guangzhou University of Chinese Medicine 
the First Affiliated Hospital of Guangzhou University of Chinese Medicine

\section{Research}

Keywords: Acute rhinitis, Surfrin ${ }^{\circledR}$ Intranasal Spray, Randomized controlled trial

Posted Date: December 16th, 2020

DOl: https://doi.org/10.21203/rs.3.rs-128423/v1

License: (c) (i) This work is licensed under a Creative Commons Attribution 4.0 International License. Read Full License 


\section{Abstract}

Background: Phospholipids form a natural defense and self-cleansing system in the nose, and the phospholipids supplemented via nasal sprays might stabilize or restore the impaired "nasal surfactant," thereby maintaining the natural moisture film, protecting and moisturizing the nasal mucosa, and facilitating mucociliary clearance. Acute rhinitis is a self-limited, prevalent human disease caused by viral infection of the upper respiratory tract and is characterized by nasal dryness, sneezing, nasal congestion and discharge. Acute rhinitis is currently treated by symptom alleviation. This study investigates the efficacy and safety of a natural phospholipid intranasal spray in attenuating the nasal discomfort of patients with acute rhinitis.

Methods: In this randomized, positive control, noninferiority trial, 144 patients with acute rhinitis were recruited by otolaryngologists in two Grade $3 \mathrm{~A}$ public and research hospitals, and randomly allocated to experimental and control groups in a 1:1 ratio to receive a natural phospholipid spray, i.e., Surfrin ${ }^{\circledR}$ Intranasal Spray (Surfrin ${ }^{\circledR}$ ), or a physiological sea water nasal spray, i.e., Bright Nose ${ }^{\circledR}$ Physiological Sea Water Nasal Spray (BNNS), respectively.

The primary outcome was the effective rate, which was judged by the nasal sign score reduction index. The secondary outcome was changes in the total scores of nasal symptoms (TNSS). Any adverse events (AEs) of the study were recorded.

Results: In the final statistical analysis, there were 68 participants in the experimental group and 71 participants in the control group. The effective rates of Surfrin ${ }^{\circledR}$ and BNNS were $98.5 \%$ and $91.5 \%$, respectively. The TNSS decreased from $5.75 \pm 1.83$ to $0.96 \pm 1.35$ in the experimental group, and from $5.65 \pm 1.48$ to $1.20 \pm 1.38$ in the control group. The experimental group had one AE not related to treatment $(1.41 \%)$, and another occurred in the control group $(1.39 \%)$ and was probably related to treatment. No serious AEs or adverse reactions occurred.

Conclusions: The results of this trial demonstrate the good safety and efficacy of the natural phospholipid Surfrin ${ }^{\circledR}$ Intranasal Spray as an alternative treatment for acute rhinitis.

Trial registration: Chinese Clinical Trial Registry, Identifier: ChiCTR1900025655, September 4, 2019, retrospectively registered).

\section{Background}

Acute rhinitis, a significant characteristic of the common cold, is a self-limited, prevalent human disease caused by viral infection of the upper respiratory tract [1]. Typical symptoms include nasal dryness, sneezing, nasal congestion and discharge [2-4]. Although the infection is often mild, it can bring a heavy burden on the cost to society [5]. The incidence of acute rhinitis varies with age. On average, children suffer 5 to 7 times and adults suffer 2 to 3 times each year [6]. At present, there is no effective medicine for acute rhinitis, so the main treatments focus on symptom relief $[2,7]$. Human rhinoviruses are the 
primary cause of nasal mucous membrane infections, which result in the impairment of epithelial integrity and a decrease in the nasal ciliary clearance rate $[8,9]$. As recommended by expert consensus [10], local nasal administration is the main treatment for acute and chronic rhinitis. Among recommended treatments, nasal saline irrigation is effective in treating various types of rhinitis and improving ciliary clearance on the mucosal barrier by liquefying nasal secretions[10, 11].

A new alternative treatment for mucosal barrier disorders is the nasal application of liposomes consisting of phospholipids. Phospholipids are components of biological cell membranes and are also found on the surface of the nasal mucosa. They make up most of the protective nasal surfactant layer $[12,13]$. Liposomes, which consist of phospholipids, can protect the nasal mucous membrane by complementing the lipid layer [14], removing antigens and improving mucociliary clearance by mechanical cleansing [13, $15]$, and aid in healing the damaged nasal mucosa by covering the surface of the mucosa $[13,16,17]$. Several studies have demonstrated the mechanism of phospholipids and shown that liposomal nasal sprays have a positive effect on relieving nasal symptoms and in treating nasal diseases associated with mucostasis $[13,18-20]$.

The present study aims to investigate the efficacy, safety and tolerability after application of a new phospholipid intranasal spray, Surfrin ${ }^{\circledR}$ Intranasal Spray (Surfrin $\AA$ ), compared with a well-known saline irrigation spray, Bright Nose® Physiological Sea Water Nasal Spray (BNNS). Both medical devices contain isotonic sprays with $\mathrm{pH}$ values similar to those of the nasal environment. Surfrin® stabilizes a natural nasal surfactant in the form of a nano microemulsion containing a higher phospholipid concentration in a smaller particle size compared to traditional liposomal sprays. As a result, lower surface tension, better stability and a good dispersion effect are obtained. It is also differentiated from the competition by its natural and safe ingredients, which are all on the Generally Recognized as Safe (GRAS) List from the U.S. Food and Drug Administration (FDA). BNNS is an over-the-counter (OTC) nasal saline irrigation medical device that has been widely used in China for many years [21-23].

\section{Methods}

\section{Study design and setting}

This is a multicenter, randomized, positive control, noninferiority study. From 24 July 2018 to 25 September 2019, a total of 144 participants were recruited from two Grade 3A public and research hospitals in Guangzhou, China: The First Affiliated Hospital of Guangzhou University of Chinese Medicine (FAHGUCM) and The Second Affiliated Hospital of Guangzhou University of Chinese Medicine (Guangdong Provincial Hospital of Chinese Medicine, GDPHCM). After obtaining written informed consent, eligible participants were randomly assigned to the experimental group or the control group in a 1:1 ratio. The study consisted of a 2-day screening period (before therapy) and a $5 \pm 2$-day treatment period. The study flowchart is shown in Fig. 1, and the study design schedule is shown in Table 1. 
Table 1

Study design schedule

\begin{tabular}{|c|c|c|c|}
\hline $\begin{array}{l}\text { Stage } \\
\text { Items }\end{array}$ & $\begin{array}{l}\text { Screening baseline } \\
(\text { day-2-0) }\end{array}$ & $\begin{array}{l}\text { 1st visit } \\
(\text { day } 3+1)\end{array}$ & $\begin{array}{l}\text { 2nd visit } \\
\text { (day } 5+2 \text { ) }\end{array}$ \\
\hline Informed Consent & 0 & & \\
\hline Eligibility Criteria & 0 & & \\
\hline Demographics & 0 & & \\
\hline Physical Examination & 0 & 0 & 0 \\
\hline Medical History & 0 & & \\
\hline Randomization & 0 & & \\
\hline Nasal Spray Dispense & 0 & & \\
\hline Nasal Symptoms Score & 0 & 0 & 0 \\
\hline Anterior Rhinoscopy & 0 & 0 & 0 \\
\hline Usage Log Dispense & 0 & & \\
\hline Usage Log Check & & 0 & 0 \\
\hline Concomitant Medication & 0 & 0 & 0 \\
\hline Adverse Events Monitoring & 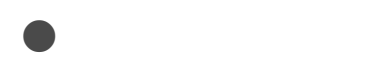 & 0 & 0 \\
\hline Usage Log Re-collect & & & 0 \\
\hline Nasal Spray Count & & & 0 \\
\hline \multicolumn{4}{|c|}{$\begin{array}{l}\text { Note: The nasal sprays and usage logs were dispensed and re-collected by the designated authorized } \\
\text { personnel. The nasal spray should have been used } 3 \text { times as required at the first visit or second visit. } \\
\text { If clinical recovery was achieved at the first visit, the nasal spray and usage logs could be re-collected, } \\
\text { the data collection and clinical study were then completed. }\end{array}$} \\
\hline
\end{tabular}

\section{Participants}

Participants were recruited via local advertisements and doctor referrals from otorhinolaryngology clinics at FAHGUCM and GDPHCM. Information about the study, processing and scheduling was carefully explained before enrollment. The participants were required to meet the Diagnostic Criteria for Acute Rhinitis [3] (Table 2). The detailed eligibility criteria are listed in Table 3 and Table 4. 
Symptoms:

Symptoms included nasal itching or dryness, sneezing, nasal obstruction, hyposmia, watery rhinorrhea, or mucoid secretions after one or two days. These symptoms may be accompanied by fever, xerostomia, pharynx dryness, headache and general discomfort.

Signs:

The nasal mucosa is congested and swollen with an increase in nasal secretions.

Table 3

Eligibility criteria for acute rhinitis participants

\section{Inclusion criteria}

In accordance with the Diagnostic Criteria for Acute Rhinitis. Onset time of less than 48 hours. Congested nasal mucosa, which was examined by an otolaryngologist using anterior rhinoscopy; nasal sign score greater than 1 (Table 4).

Age 18 to 65 years old, no sex limitation.

Participants provided written informed consent and volunteered to participate in the study.

\section{Exclusion criteria}

Acute rhinitis caused by specific inflammations including allergies, influenza, measles, scarlet fever, and chickenpox, etc.

Participants with high fever (axillary temperature of over $38^{\circ} \mathrm{C}$ ).

Participants with tracheitis, bronchitis and sinusitis or other diseases.

Participants with severe primary diseases, such as cardiovascular, cerebrovascular, liver, kidney and hematopoietic system diseases, or with mental illness.

Participants with severe structural obstruction of the nose (such as nasal septum deviation, nasal polyps or a volume of adenoid hypertrophy of over $75 \%$ ).

Pregnant or lactating women and those who were unable to use contraceptive measures as required during the study.

Participants allergic to the interventions.

Participants who had received systemic steroids, antihistamines or intranasal medication in the previous one week.

- Individuals who had participated in clinical research in the past three months.

- Individuals who were considered not suitable for participating in this study by the investigators. 
Table 4

Nasal sign scores

None (score: 0) Normal signs

Mild (score: 1) Extensive congestion and dryness of the nasal mucosa

Moderate (score: 2)

3)
Swollen nasal mucosa with watery secretions in the general nasal meatus or the nasal floor

Swollen nasal mucosa with mucoid secretions in the general nasal meatus or the nasal floor

\section{Randomization and allocation concealment}

This was a randomized, unblinded clinical trial. Randomization was formulated and performed by the Key Unit of Methodology in Clinical Research of GDPHCM. SAS software version 9.2 (SAS Institute Inc., Cary, USA) was used to generate random numbers. One random number and its corresponding group were then enclosed in each opaque envelope. The outside of each envelope was sealed and labeled with the study name, hospital name, and patient registration sequence number and then kept in a locked cabinet. Only authorized and trained investigators were permitted to take out and distribute the envelopes. Once an eligible participant signed a written informed consent form, an investigator applied an envelope according to the patient's number in the enrollment sequence. The eligible participant was then assigned to the group indicated inside the envelope.

\section{Interventions}

The participants in the experimental group were given Surfrin ${ }^{\circledR}$ manufactured by Shenzhen JSK Consumer Healthcare, Ltd. (Shenzhen, Guangdong Province, China) (medical device registration certificate number: YueXieZhuZhun20192140960; batch number: 1712001). The participants in the control group were given BNNS made by Zhejiang Langke Bio-Engineering Co., Ltd. (Hangzhou, Zhejiang Province, China) (medical device registration certificate number: ZheXieZhuZhun20172660882; batch number: 171205). All nasal sprays were produced by the manufacturers complying with Good Manufacturing Practice. Two follow-up visits were performed during the treatment period. No other treatment for acute rhinitis was allowed for any participant in either group during the study period. If a participant's symptoms and signs had disappeared at the first visit and achieved clinical recovery, the study could be ended in advance; otherwise, the study was terminated after the second visit. If a participant was told to finish the study, the designated authorized person re-collected the device and measured the residual amount with a disposable syringe to obtain the actual residual amount. The compliance of patients was judged by the total amount of Surfrin $\circledast$ and BNNS, the amount of each spray, the total days of use, the times of daily use and the residual amount. According to the information in the package leaflet, the detailed user instructions for the two intervention devices are presented in Table 5a-b and Fig. 2. 
Step 1: Shake for five seconds to ensure that there are no loose parts and that the solution in the spray bottle is mixed evenly (Fig. 2A).

Step 2: Take off the protective cover and safety circlip (Fig. 2B).

Step 3: Before the product is used for the first time, hold the spray bottle with the middle finger, ring finger and pinky finger, with the thumb and index finger clamping the spray pump, and press the spray pump to release one spray, and then release three sprays to the air. Now the product can be used (Fig. 2C).

Step 4: Place the nozzle slightly deeper into the nasal cavity and slightly pointed to the rear, covering the other nostril with fingers (Fig. 2D).

Step 5: Hold your breath and press the spray pump.

Step 6: Remove the nozzle.

Step 7: Repeat steps 4 to 6 for the other nasal cavity. Use three sprays in each nostril, three times daily.

Step 8: Release one spray to the air after use, and then replace the protective cover and safety circlip.

Table 5b

User instructions for BNNS

Step 1: Open the blue dust cover and blue safety buckle.

Step 2: Place the nozzle into the nasal cavity and keep it consistent with the direction of the nostril.

Step 3: Keep your head upright.

Step 4: Gently press the spray pump to evenly eject the liquid and distribute it in the nasal cavity.

Step 5: Use four sprays for each nostril, three times daily.

Step 6: Blow out the washing liquid after pressed.

\section{Outcome measurement}

The primary outcome was the effective rate, which is the sum of the percentage of the total number of healed cases, markedly effective cases and effective cases in each group. The effective rate was judged by the nasal sign score reduction index (SSRI). The nasal signs were examined by an otolaryngologist using anterior rhinoscopy, and the nasal sign scores are shown in Table 4. The SSRI was calculated as follows: SSRI= [(pretreatment score-post-treatment score)/pretreatment score $] \times 100 \%$. SSRI $=100 \%$ means healed, $60 \% \leq \mathrm{SSRI}<100 \%$ means markedly effective, $30 \% \leq \mathrm{SSRI}<60 \%$ means effective, $0<\mathrm{SSRI}$ $<30 \%$ means slightly effective, and SSRI $\leq 0$ means ineffective. The secondary outcome measure was 
the change in the total nasal symptom score (TNSS, including sneezing, rhinorrhea, congestion and dryness) (Table 6).

As safety outcomes, adverse events (AEs) and adverse drug reactions (ADRs) were recorded in the case report form (CRF), including the occurrence time, severity, management and causality from the trial. The severity of the AEs and ADRs and the relationship between the trial and AEs were assessed according to the Standardized Case Causality Assessment of the WHO Uppsala Monitoring Centre System [24].

Table 6

Nasal symptom scores

\begin{tabular}{|ll|}
\hline None (score: 0 ) & No symptoms \\
\hline Mild (score: 1 ) & Symptoms are slight and tolerable \\
\hline Moderate (score: 2 ) & Symptoms are troubling, but tolerable \\
\hline Severe (score: 3 ) & Symptoms are intolerable and interfere with daily life and/or sleep \\
\hline
\end{tabular}

\section{Data collection and management}

All information from the CRFs was carefully recorded. All errors needed to be crossed out and corrected, after which the correction must be explained, signed and dated by the investigator. Patient withdrawals and/or missed follow-up visits needed to be recorded in the CRFs. All nasal sprays were kept in each hospital's Good Clinical Practice (GCP) pharmacy, and a designated authorized and trained pharmacist in each pharmacy was assigned to be responsible for nasal spray distribution and recording. The participants were required to bring the remaining intranasal spray during follow-up, and research assistants used a disposable syringe $(20 \mathrm{ml})$ to measure the remaining amount. There was an acceptable level of "compliance" in the data for the remaining amount (Table 7).

Table 7

Compliance acceptance scope

\begin{tabular}{|c|c|c|c|}
\hline & & Experimental Group & Control Group \\
\hline Capacity & & $15 \mathrm{~mL}$ & $25 \mathrm{~mL}$ \\
\hline \multirow[t]{3}{*}{ Compliance Acceptable residue } & Day Three & $\leq 11.4 \mathrm{~mL}$ & $\leq 17.8 \mathrm{~mL}$ \\
\hline & Day Four & $\leq 10.2 \mathrm{~mL}$ & $\leq 15.4 \mathrm{~mL}$ \\
\hline & Day Five & $\leq 9 \mathrm{~mL}$ & $\leq 13 \mathrm{~mL}$ \\
\hline
\end{tabular}

If the residue amount met the above conditions, the subject's compliance was judged to be good. If the residue amount did not meet the above conditions, the subject's compliance was judged to be poor. 
The data were entered using the double-entry method. The research assistants double checked the data before logging them and promptly notified the research team if any discrepancies were found. All modifications were marked on the CRFs. The database was locked after all data were cleaned. If a participant withdrew from the study either during the treatment period or the follow-up phase, the reasons were clarified and statistically analyzed.

\section{Sample size calculation}

This was a randomized, noninferiority test study focused on determining the efficacy of Surfrin ${ }^{\circledR}$ for acute rhinitis. The primary outcome was the effective rate. According to a clinical literature report $[25,26]$, the noninferiority boundary value is $\delta=0.15$. With reference to the "Guidelines for the Design of Clinical Trials for Medical Devices [27]" and the use of PASS (version 11.0) software for calculations, it was found that each group needs at least 49 cases. The final number of cases with complete data collection was determined to be not less than 60 in each group, with a total of 120 cases. Considering a $20 \%$ dropout rate, 144 cases were needed, with 72 cases in each group. In this study, 144 patients were recruited from the two hospitals, with 72 participants from each hospital.

\section{Data analysis}

The measurement data are expressed as the mean, median, minimum, and maximum values. Two-by-two chi square tests (or accurate probability methods) and confidence interval methods of non-inferiority tests were used for the comparison of the total effective rate among the groups. If the lower limit of the $97.5 \%$ confidence interval on one side was greater than the non-inferiority boundary value, non-inferiority was established. The rank sum test was used to compare rank data. The paired t-test (or Wilcoxon signed-rank test) was used for self-comparison in the group. Two independent sample t-tests (including $95 \%$ confidence interval calculation) were used for intergroup comparisons, and the rank sum test was used for variables with a nonnormal distribution and uneven variance. Efficacy analyses were carried out on the intent-to-treat (ITT) population. All of the data were analyzed using Statistical Product and Service Solutions (SPSS) (SPSS Inc., Chicago, IL, USA; version 17.0) and STATA software 11.0/SE (Stata Corp. College Station, Texas, USA).

\section{Results}

There were 68 participants ( 27 male and 41 female) with a mean age of 27.57 years (standard deviation 8.60 ) in the experimental group, and 71 participants ( 23 male and 48 female) with a mean age of 29.65 years (standard deviation 11.90) in the control group (Fig. 1, Table 8). There were no significant differences in the demographic characteristics of the groups. There were also no significant differences in the axillary temperature, anterior rhinoscopy score, drug allergy history, operation history, or other medical history of the groups (Table 8). The intervention was initiated after all cases were randomized. 
Table 8

Baseline characteristics of the study participants

\begin{tabular}{|c|c|c|c|c|c|}
\hline Item & Classification & $\begin{array}{l}\text { Experimental } \\
\text { Group } \\
(n=68)\end{array}$ & $\begin{array}{l}\text { Control } \\
\text { Group } \\
(n=71)\end{array}$ & 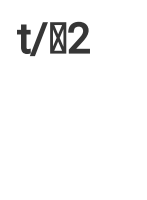 & $P$ \\
\hline \multicolumn{6}{|c|}{ - Baseline demographic features } \\
\hline Age & $\begin{array}{l}\text { (mean } \pm S D \\
\text { years) }\end{array}$ & $27.57 \pm 8.60$ & $29.65 \pm 11.90$ & -1.174 & 0.243 \\
\hline \multirow[t]{2}{*}{ Sex } & Male & $27(39.7 \%)$ & $23(32.4 \%)$ & \multirow[t]{2}{*}{0.806} & \multirow[t]{2}{*}{0.369} \\
\hline & Female & $41(60.3 \%)$ & $48(67.6 \%)$ & & \\
\hline \multicolumn{6}{|c|}{ - Examination results before treatment } \\
\hline Axillary temperature & $\left(\right.$ mean $\left.\pm S D,{ }^{\circ} \mathrm{C}\right)$ & $36.6 \pm 0.29$ & $36.6 \pm 0.26$ & 0.752 & 0.453 \\
\hline \multirow[t]{2}{*}{ Anterior rhinoscopy } & Moderate & $59(86.8 \%)$ & $59(83.1 \%)$ & \multirow[t]{2}{*}{0.364} & \multirow[t]{2}{*}{0.546} \\
\hline & Severe & $9(13.2 \%)$ & $12(16.9 \%)$ & & \\
\hline \multicolumn{6}{|l|}{ Disease history } \\
\hline \multirow[t]{2}{*}{ Drug allergy history } & No & $66(97.1 \%)$ & $69(97.2 \%)$ & \multirow[t]{2}{*}{-} & \multirow[t]{2}{*}{$1.000^{*}$} \\
\hline & Yes & $2(2.9 \%)$ & $2(2.8 \%)$ & & \\
\hline \multirow{2}{*}{$\begin{array}{l}\text { Past surgical } \\
\text { history }\end{array}$} & No & $63(92.6 \%)$ & $69(97.2 \%)$ & \multirow[t]{2}{*}{-} & \multirow[t]{2}{*}{$0.268^{*}$} \\
\hline & Yes & $5(7.4 \%)$ & $2(2.8 \%)$ & & \\
\hline \multirow[t]{2}{*}{ Others } & No & $53(77.9 \%)$ & $52(73.2 \%)$ & \multirow[t]{2}{*}{0.416} & \multirow[t]{2}{*}{0.519} \\
\hline & Yes & 15 (22.1\%) & 19 (26.8\%) & & \\
\hline
\end{tabular}

\section{Effective rate}

After the treatment, according to the SSRI, the result of ITT analysis revealed that there were $47(69.1 \%)$ markedly effective cases, $20(29.4 \%)$ effective cases, $0(0 \%)$ weakly effective cases, and $1(1.5 \%)$ ineffective case in the experimental group, while in the control group, there were 39 (54.9\%) markedly effective cases, 26 (36.6\%) effective cases, 0 (0\%) weakly effective cases, and $6(8.5 \%)$ ineffective cases, with no significant difference between the two groups (Table 9). The effective rate was $98.5 \%$ in the experimental group and $91.5 \%$ in the control group. The difference in the effective rate was $7.0 \%$, and the lower limit of the unilateral $97.5 \%$ confidence interval was $-2.6 \%$, which is greater than $-15 \%$, indicating 
that the effective rate of the treatment group was not inferior to that of the control group (Table 10). The risk ratio for the effective rate was 1.089 (95\% Cl: 1-1.16), which suggests that there was no difference between two interventions for improving nasal sign.

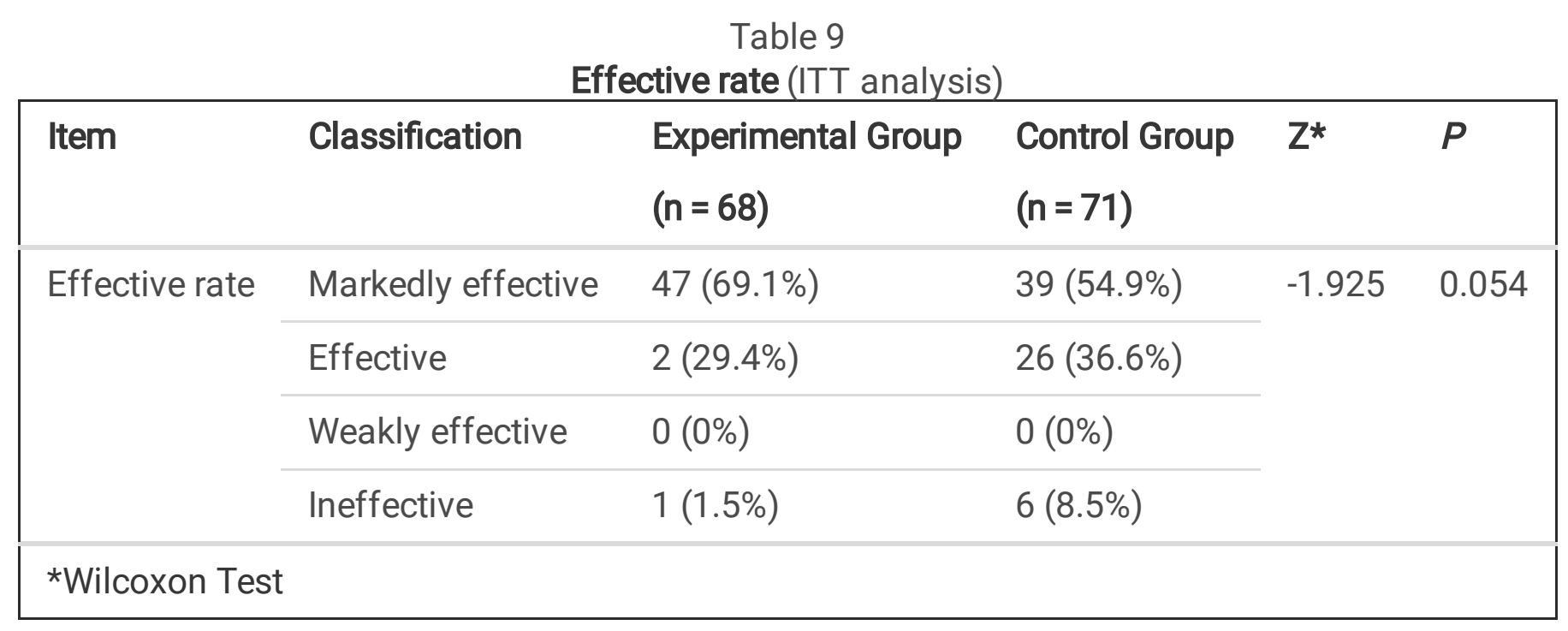

Table 10

Noninferiority test of the effective rate (ITT analysis)

\begin{tabular}{|c|c|c|c|c|c|c|}
\hline Classification & $\begin{array}{l}\text { Experimental } \\
\text { Group } \\
(n=68)\end{array}$ & $\begin{array}{l}\text { Control } \\
\text { Group } \\
(n=71)\end{array}$ & $\begin{array}{l}\text { Rate } \\
\text { difference }\end{array}$ & $\begin{array}{l}97.5 \% \mathrm{Cl} \\
\text { lower limit }\end{array}$ & $\begin{array}{l}\text { Risk } \\
\text { ratio }\end{array}$ & $\begin{array}{l}\text { Difference } \\
(95 \% \mathrm{Cl})\end{array}$ \\
\hline Effective & 67 (98.5\%) & $\begin{array}{l}65 \\
(91.5 \%)\end{array}$ & $7.0 \%$ & $-2.6 \%$ & 1.08 & $(1,1.16)$ \\
\hline Ineffective & $1(1.5 \%)$ & $6(8.5 \%)$ & & & & \\
\hline
\end{tabular}

\section{TNSS}

The results of ITT analysis revealed that there were no significant differences in the TNSS between the two groups before and after treatment, and the TNSS decreased for both groups after treatment: for the experimental group, the TNSS decreased from $5.75 \pm 1.83$ to $0.96 \pm 1.35$ and for the control group from $5.65 \pm 1.48$ to $1.20 \pm 1.38$ (Table 11). The separate analysis results of all four nasal symptoms are shown in the Supplementary material. 
Table 11

Comparison of total nasal symptom scores between the two groups (ITT analysis)

\begin{tabular}{|c|c|c|c|c|c|c|}
\hline Item & Group & $\mathbf{N}$ & $\overline{\mathrm{x}} \pm \mathrm{s}$ & Difference $(95 \% \mathrm{Cl})$ & $\mathrm{t}$ & $\mathrm{p}$ \\
\hline \multirow[t]{2}{*}{ Baseline } & Experimental & 68 & $5.75 \pm 1.83$ & \multirow[t]{2}{*}{$0.10(-0.46,0.66)$} & \multirow[t]{2}{*}{0.362} & \multirow[t]{2}{*}{0.718} \\
\hline & Control & 71 & $5.65 \pm 1.48$ & & & \\
\hline \multirow[t]{2}{*}{ V1 } & Experimental & 68 & $2.59 \pm 1.75$ & \multirow[t]{2}{*}{$-0.12(-0.71,0.48)$} & \multirow[t]{2}{*}{-0.384} & \multirow[t]{2}{*}{0.701} \\
\hline & Control & 71 & $2.70 \pm 1.81$ & & & \\
\hline \multirow[t]{2}{*}{ V2 } & Experimental & 61 & $1.05 \pm 1.40$ & \multirow[t]{2}{*}{$-0.26(-0.76,0.23)$} & \multirow[t]{2}{*}{-1.052} & \multirow[t]{2}{*}{0.295} \\
\hline & Control & 64 & $1.31 \pm 1.40$ & & & \\
\hline \multirow{2}{*}{$\begin{array}{l}\text { V2 } \\
\text { (Data filling*) }\end{array}$} & Experimental & 68 & $0.96 \pm 1.35$ & \multirow[t]{2}{*}{$-0.24(-0.70,0.21)$} & \multirow[t]{2}{*}{-1.040} & \multirow[t]{2}{*}{0.300} \\
\hline & Control & 71 & $1.20 \pm 1.38$ & & & \\
\hline \multirow{2}{*}{$\begin{array}{l}\text { V2-baseline } \\
\text { (Data filling*) }\end{array}$} & Experimental & 68 & $-4.79 \pm 2.25$ & \multirow[t]{2}{*}{$-0.34(-1.04,0.35)$} & \multirow[t]{2}{*}{-0.975} & \multirow[t]{2}{*}{0.331} \\
\hline & Control & 71 & $-4.45 \pm 1.90$ & & & \\
\hline
\end{tabular}

\section{Safety}

Both treatments were well tolerated. Two AEs, one back pain in the experimental group and one vestibulitis in the control group, were reported, with incidences of $1.41 \%$ and $1.39 \%$, respectively. None of the AEs were serious, and these two participants kept using the nasal spray. Back pain was not considered to be related to the nasal spray, and it was relieved after Chinese herbal treatment. Vestibulitis in the control group may be related to treatment and was ameliorated without any treatment.

\section{Discussion}

\section{Effectiveness and safety of Surfrin ${ }^{\circledR}$}

Acute rhinitis is a self-limited disease, and current treatments for acute rhinitis mainly focus on symptom relief. Topical treatment with a nasal spray is recommended by the Expert Census of Rhinitis [10]. The results of this 5 -day treatment for acute rhinitis showed that the effective rate was $98.5 \%$ in the Surfrin $\circledast$ group and $91.5 \%$ in the BNNS group, achieving the primary outcome. The results of the ITT analysis revealed that the effective rate in the Surfrin $\AA$ group was not inferior to that in the BNNS group. With respect to symptom reduction, TNSS decreased in both groups after treatment: from $5.75 \pm 1.83$ to $0.96 \pm$ 1.35 in the Surfrin ${ }^{\circledR}$ group and from $5.65 \pm 1.48$ to $1.20 \pm 1.38$ in the BNNS group. The difference between these values after treatment was not statistically significant. The improvements achieved in the effective 
rate and symptom reduction showed a therapeutic effect of Surfrin ${ }^{\circledR}$ Intranasal Spray for acute rhinitis as well as the BNNS.

A previous study with a phospholipid nasal spray demonstrated efficacy in reducing nasal symptoms in patients suffering from rhinitis sicca comparable to that of generally recognized treatment with dexpanthenol nasal ointment and saline nasal spray [20]. In patients with seasonal-allergicrhinoconjunctivitis, compared to standard therapy with glucocorticoids or antihistamines, liposomal nasal spray demonstrated good tolerability and a significant reduction in nasal and conjunctivitis symptoms [13]. A single-blind, placebo-controlled, crossover design study demonstrated that an intranasal microemulsion treatment with a specific lipid-based composition attenuated allergen challenge-induced nasal symptoms and plasma exudation in allergic rhinitis [18]. The results of a singlecenter, prospective, controlled, open, noninterventional study showed that treatment of chronic rhinosinusitis with a liposomal nasal spray reduced nasal symptoms and improved quality of life significantly as a guideline-recommended treatment [19]. Taken together, these earlier studies and the present study indicate that phospholipid nasal sprays are an effective and comparable alternative to guideline-recommended therapy for inflammatory diseases of the nose and rhinosinusitis, especially for nasal symptom reduction.

With respect to safety outcome, two AEs occurred: one report of back pain in the experimental group and one report of vestibulitis in the control group. The incidence of AEs was low, and no serious AEs occurred. The adverse event in the experimental group was considered to be unrelated to the device, which demonstrates that Surfrin ${ }^{\circledR}$ was safe and well tolerated.

\section{Potential mechanisms of Surfrin®}

Mucosal barrier disorders are important pathologic features of acute rhinitis and other upper respiratory inflammation diseases. Surfrin ${ }^{\circledR}$ consists of a higher phospholipid concentration in the form of a nano microemulsion in a smaller particle size. Phospholipids are components of biological cell membranes and make up $75.35 \%$ of the protective nasal surfactant layer. They easily fuse with the cell membrane of the nasal mucosa to form a natural defense [12]. Phospholipid surfactants are beneficial for reducing the surface tension at the nasal air-mucus interface and lubricating the nasal cavity, increasing the ciliary beat frequency, reducing the adhesion of pathogenic microorganisms or allergens, stabilizing the barrier of the respiratory tract and enhancing the anti-inflammatory and wound-healing capacities of the upper airway mucosa, including the nasal mucosa [12-17].

\section{Limitations of this study}

There are limitations to this study. The first limitation is the lack of blinding of participants, personnel and outcome measures, which may lead to performance bias or detection bias. The second limitation is the lack of internationally validated outcome measures to evaluate acute rhinitis. To our knowledge, there is no well-acknowledged outcome measure for evaluating acute rhinitis. In this study, the "effective rate" assessed the proportion of participants who achieved certain improvement in nasal signs and was 
evaluated by an otolaryngologist, while TNSS assessed the improvement of nasal symptoms and was evaluated by participants. Both outcome measures reflected overall improvement, but the analysis would be more comprehensive if there were an internationally validated quality-of-life scale. The third limitation is that the sample size calculation was based on a previous study [24, 25]; thus, the sample size of the study might be different if more cases had been observed by a rigorous RCT. The last limitation is that the noninferiority boundary value $(\delta=0.15)$ was a little large, which was determined by the data of previous studies $[25,26]$, and the result of this clinical trial shows that even if the noninferiority threshold is set at 0.05 , the hypothesis of noninferiority is still valid.

\section{Conclusion}

Treatment of acute rhinitis with Surfrin ${ }^{\circledR}$ Intranasal Spray is effective and well-accepted by patients and has a positive safety profile in terms of side effects. This study indicated that Surfrin ${ }^{\circledR}$ is comparable to BNNS in alleviating acute rhinitis symptoms and nasal signs. Surfrin ${ }^{\circledR}$ offers a good alternative therapy for acute rhinitis patients considering its safety and efficacy.

\section{Abbreviations}

ADRs: Adverse Drug Reactions

AEs: Adverse Events

BNNS: Bright Nose® Physiological Sea Water Nasal Spray

CRF: Case Report Form

FAHGUCM: The First Affiliated Hospital of Guangzhou University of Chinese Medicine

FDA: U.S. Food and Drug Administration

GDPHCM: Guangdong Provincial Hospital of Chinese Medicine (The Second Affiliated Hospital of Guangzhou University of Chinese Medicine)

GCP: Good Clinical Practice

GRAS: Generally Recognized as Safe

ITT: Intent-to-treat

OTC: Over-the-counter

SPSS: Statistical Product and Service Solutions

SSRI: Sign Score Reduction Index 
Surfrin ${ }^{\circledR}$ : Surfrin ${ }^{\circledR}$ Intranasal Spray

TNSS: Total Nasal Symptom Score

\section{Declarations}

\section{Acknowledgments}

We would like to acknowledge the doctors who helped screen and enroll eligible patients, including Renliang Zhu, Caifeng Chen, Jianwen Xiang, Lanfang Li, Zhe Kong, and Shaoqing Liu in the Otorhinolaryngology Department of GDPHCM. We would also like to acknowledge the statisticians, including Geng Li and Zewei Wen at the Key Unit of Methodology in Clinical Research and GDPHCM, who contributed greatly to the data analysis. We thank Xun Zhang and Meijun Kong at the Drug Clinical Trial Institution of GDPHCM, Yanping Du and Tianhui Yuan at the Drug Clinical Trial Institution of FAHGUCM, and Minmin Liu and Liming Xie from JSK Consumer Healthcare, Ltd., for supervising the study. We also thank Minyi Liu and Zhiyue Deng in the pharmacy of GDPHCM and Zhongwen Chen in the pharmacy of FAHGUCM for checking and distributing the study medical devices.

\section{Funding}

This study was sponsored by JSK Consumer Healthcare, Ltd., and their contact information is as follows: JSK Consumer Healthcare, Ltd.; 18B-102, Zhonghaixin , Ganli 2nd Road, Longgang District, Shenzhen, 518112, China; info@hightidetx.com. This study was supervised by the Drug Clinical Trial Institutions of GDPHCM and FAHGUCM, ensuring that the study was conducted under GCP; their contact information is as follows: Drug Clinical Trial Institution of GuangDong Provincial Hospital of Chinese Medicine, 111 Dade Road, Yuexiu District, Guangzhou, 510120, China; gcpbgs@gzucm.edu.cn; Drug Clinical Trial Institution of the First Affiliated Hospital of Guangzhou University of Chinese Medicine, 16 Jichang Road, Baiyun District, Guangzhou, Guangdong Province, 510405, China; gzygcp@126.com. The study design, data collection, or preparation of the manuscript for publication was implemented by the Otorhinolaryngology Department in GDPHCM and FAHGUCM. The principal investigators of this study are Professor Yunying Li, Professor Jiyan Xia and Professor Yan Ruan. Yunying Li and Jiyan Xia are clinical doctors at the Otorhinolaryngology Department in GDPHCM, and Yan Ruan is a clinical doctor at the Otorhinolaryngology Department in FAHGUCM. They have no conflicts of interest with regard to JSK Consumer Healthcare, Ltd.

\section{Availability of data and materials}

The data that support the findings of this study are available from JSK Consumer Healthcare, Ltd. but restrictions apply to the availability of these data, which were used under license for the current study, 
and so are not publicly available. Data are however available from the authors upon reasonable request and with permission of JSK Consumer Healthcare, Ltd.

\section{Authors' contributions}

$Y Y L, J Y X$ and YR contributed to the design of the trial. WWOY was involved in designing the statistical methods used in the study. JYX, QLL, PYW, HBZ, JHW, YL, CSL, YFW, CQL, WYC, YYL and YR participated in the project development and collected the outcome data. JYX, QLL, WH and XCF prepared the initial draft of the manuscript. HBZ, PYW and WWOY were involved in the data management and statistical analysis. JYX, YYL and YR led the project. All authors were responsible for drafting the manuscript and approved the final version.

\section{Ethics approval and consent to participate}

Ethics approval for this trial was obtained from the Ethics Committees of GDPHCM (number: B2018-07002) and FAHGUCM (number: ZYYEC [2018]008). All participants were provided with sufficient time to consider whether to enter the trial or not. The investigators and research assistants obtained written informed consent from each participant before randomization. The study was conducted in accordance with the ethical principles of the Declaration of Helsinki (2013 version).

\section{Competing interests}

The authors declare that they have no competing interests.

\section{Consent for publication}

Not applicable.

\section{References}

1. Allan GM, Arroll B. Prevention and treatment of the common cold: making sense of the evidence. CMAJ. 2014;186(3):190-199.

2. Heikkinen T, Järvinen A. The common cold. Lancet. 2003;361(9351):51-59.

3. National Health Commission of the People's Republic of China. Guiding principles for clinical research of new traditional Chinese medicine (Volume 3, 1997). Beijing, 1997:164-165. [Monograph in Chinese].

4. Kong W, Zhou L. Otorhinolaryngology Head and Neck Surgery, 3rd Edition. Beijing: People's Medical Publishing House. 2015:287-288. [Monograph in Chinese]. 
5. Bertino JS. Cost burden of viral respiratory infections: Issues for formulary decision makers. Am J Med. 2002;112 Suppl 6A:42S-49S.

6. Turner RB. Epidemiology, Pathogenesis, and Treatment of the Common Cold. Ann Allergy Asthma Immunol. 1997;78(6):531-540.

7. Passioti M, Maggina P, Megremis S, Papadopoulos NG. The common cold: potential for future prevention or cure. Curr Allergy Asthma Rep. 2014;14(2):413.

8. Waltl EE, Selb R, Eckl-Dorna J, et al. Betamethasone prevents human rhinovirus- and cigarette smokeinduced loss of respiratory epithelial barrier function. Sci Rep. 2018;8(1):9688.

9. Doyle WJ, Skoner DP, Fireman P, et al. Rhinovirus 39 infection in allergic and nonallergic subjects. J Allergy Clin Immunol. 1992;89(5):968-978.

10. Li H, Wang $X$, Wang $H$, et al. Expert consensus on classification, diagonisis and intranasal medication of rhinitis. Chin J Otorhinolaryngol Skull Base Surg. 2019;25(6):573-577. [Article in Chinese].

11. Bastier PL, Lechot A, Bordenave L, Durand M, de Gabory L. Nasal irrigation: From empiricism to evidence-based medicine. A review. Eur Ann Otorhinolaryngol Head Neck Dis. 2015;132(5):281-285.

12. Sayed RH, Abou-Elhamd KE, Abdel-Kader M, Saleem TH. Study of surfactant level in cases of primary atrophic rhinitis. J Laryngol Otol. 2000;114(4):254-259.

13. Böhm M, Avgitidou G, El Hassan E, Mösges R. Liposomes: a new non-pharmacological therapy concept for seasonal-allergic-rhinoconjunctivitis. Eur Arch Otorhinolaryngol. 2012;269(2):495-502.

14. Heiser C, Hofauer B, Scherer E, et al. Liposomal treatment of xerostomia, odor, and taste abnormalities in patients with head and neck cancer. Head \& Neck 2016;38(S1):E1232-E1237.

15. Lauriello M, Di Rubbo V, Sinatti G, et al. Correlation between SNOT-22, nasal cytology, and mood disorders in patients with allergic rhinitis treated with a liposomal nasal spray. Allergy \&Rhinology (Providence, R.I). 2019;10:2152656719866809.

16. Wang W, Lu K J, Yu C H, et al. Nano-drug delivery systems in wound treatment and skin regeneration. J Nanobiotechnology. 2019;17(1):82.

17. Ricciardiello F, Cantone $E, A$ Abate $T$, et al. Effect of liposomes, vitamins $A$ and $E$ nasal spray in reducing the healing time after septoplasty with concurrent turbinate surgery in adolescents. Minerva Pediatrica. 2017;69(1):15-21.

18. Andersson M, Greiff L, Wollmer P. Nasal treatment with a microemulsion reduces allergen challengeinduced symptoms and signs of allergic rhinitis. Acta Otolaryngol. 2008;128(6):666-669.

19. Eitenmüller A, Piano L, Böhm M, et al. Liposomal Nasal Spray versus Guideline-Recommended Steroid Nasal Spray in Patients with Chronic Rhinosinusitis: A Comparison of Tolerability and Quality of Life. J Allergy (Cairo). 2014; 146280.

20. Hahn C, Böhm M, Allekotte S, Mösges R. Tolerability and effects on quality of life of liposomal nasal spray treatment compared to nasal ointment containing dexpanthenol or isotonic $\mathrm{NaCl}$ spray in patients with rhinitis sicca. Eur Arch Otorhinolaryngol. 2013;270(9):2465-2472. 
21. He D, Zhao S, Huang $Y$, et al. Bright Nose ${ }^{\circledR}$ nasal spray in the treatment of acute rhinitis in infants and children. Chinese Journal of Otorhinolaryngology in Integrative Medicine. 2010;18 (5): 292-297. [Article in Chinese].

22. Huang $M$, Zhao $S$, Tao $L$, et al. Efficacy of Bright nose ${ }^{\circledR}$ nasal spray combined with loratadine in the treatment of allergic rhinitis in children. The Journal of Practical Medicine. 2012;28 (14): 2431-2432. [Article in Chinese].

23. Gan L, Lu L. Application of Bright nose® nasal spray in nasal irrigation after sinusitis surgery. Chinese Journal of Clinical Rational Drug Use. 2012;5(7):127. [Article in Chinese].

24. World Health Organization. The use of the WHO-UMC system for standardised case causality assessment.2018. https://www.who-umc.org/media/164200/who-umc-causality-assessment_newlogo.pdf. Access 2 June, 2020.

25. Sun $\mathrm{H}$. Clinical effect of Nuosiqing physiological seawater nasal nursing sprayer on nasal diseases. China Journal of Pharmaceutical Economics. 2014; 9(9):110-111. [Article in Chinese].

26. He D, Zhao S, Huang $Y$, et al. Observation on the efficacy of Bright Nose ® physiological sea water nasal spray in the treatment of infantile acute rhinitis .Chinese Journal of Otorhinolaryngology in Integrative Medicine.2010;18(5):292-297. [Article in Chinese].

27. Guidelines for the Design of Clinical Trials for Medical Devices. Center for Medical Device Evaluation, National Medical Production Administration. 2018. https://www.cmde.org.cn/CL0112/6937.html, Access 12 June 2020.

\section{Figures}




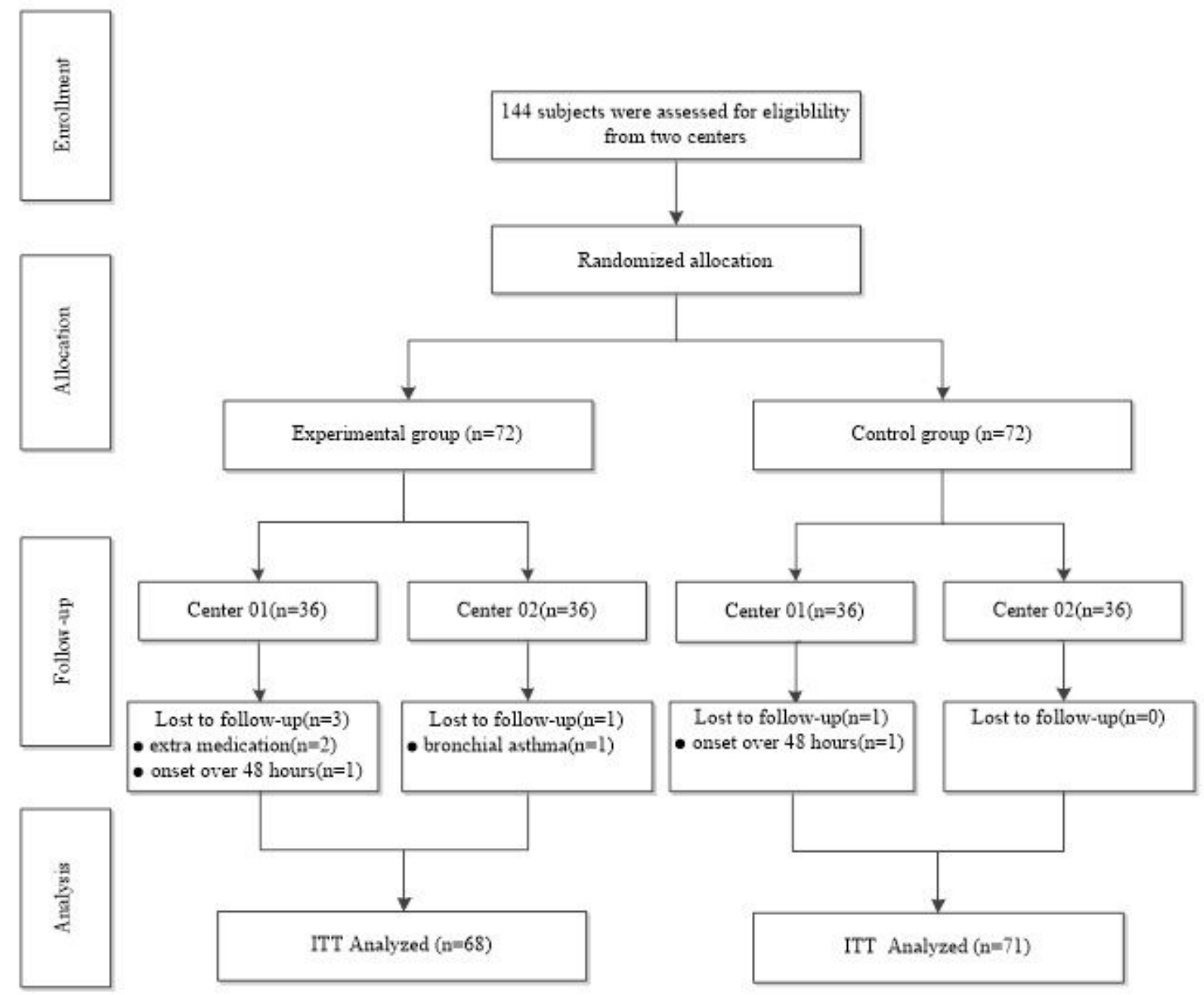

Note: Center 01: The Second Affiliated Hospital of Guangzhou University of Chinese Medicine (Guangdong Provincial Hospital of Chinese Medicine); Ceater 02. The Fust Affiliated Hospital of Guangzhou University of Chinese Medicine.

\section{Figure 1}

\section{Study flow chart}




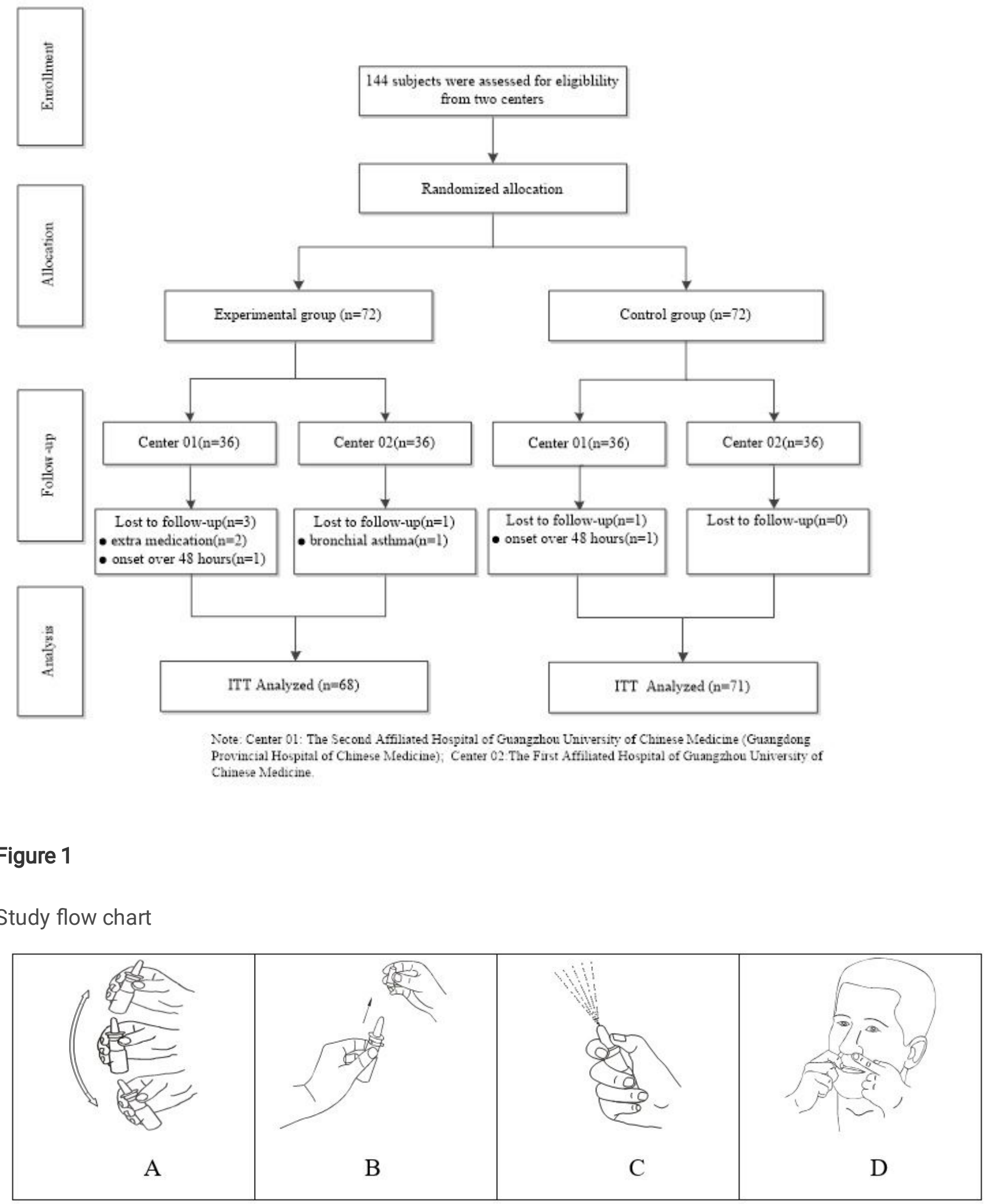

Figure 2 


\begin{tabular}{|c|c|c|c|}
\hline & & \\
\hline
\end{tabular}

Figure 2

User instructions for Surfrin ${ }^{\circledR}$

\section{Supplementary Files}

This is a list of supplementary files associated with this preprint. Click to download.

- Supplementarymaterial.docx

- Supplementarymaterial.docx

- CONSORTExtensionNPT2017Checklist.docx

- CONSORTExtensionNPT2017Checklist.docx

- CONSORTExtensionNPT2017AbstractsChecklist.docx

- CONSORTExtensionNPT2017AbstractsChecklist.docx 